\title{
Effect of purified condensed tannins from pine bark on larval motility, egg hatching and larval development of Teladorsagia circumcincta and Trichostrongylus colubriformis (Nematoda: Trichostrongylidae)
}

\author{
Abdul-Lateef Molan \\ Department of Biology, College of Sciences, Diyala University, Diyala, Iraq
}

\begin{abstract}
The effects of condensed tannins (CTs) extracted from pine bark on egg hatching, larval development and the viability of infective L3 larvae of Trichostrongylus colubriformis (Giles, 1892) and Teladorsagia circumcincta (Stadelmann, 1894) (syn. Ostertagia circumcincta) were evaluated using in vitro bioassays. Significant inhibitory effects of CTs were obtained on the viability of the infective larvae, egg hatching and larval development of both nematodes. In all bioassays, the larval stages of Te. circumcincta were significantly $(\mathrm{P}<0.05)$ more susceptible to the inhibitory effects of CT than those of Tr. colubriformis. At $1000 \mu \mathrm{g} / \mathrm{ml}, \mathrm{CTs}$ from pine bark inhibited $48 \%$ and $69 \%$ of the infective larvae of Tr. colubriformis and Te. circumcincta, respectively, from passing through the sieve relative to the control incubations (no CT added; $\mathrm{P}<0.0001$ ). At the same concentration, CTs were able to inhibit $36 \%$ and $47 \%$ of the eggs of the two parasites, respectively, from hatching relative to the control incubations without CTs. Moreover, at $150 \mu \mathrm{g} / \mathrm{ml}$, the CTs were able to inhibit $88 \%$ and $95 \%$ ( $(\mathrm{P}<0.0001$ relative to control incubation) of L1 larvae of the two nematodes, respectively, from attaining the full development to L3 larvae in comparison with the control incubations without CTs. At $200 \mu \mathrm{g} / \mathrm{ml}$, CTs were able to inhibit completely the larval development in both nematodes. Addition of $2 \mu \mathrm{g}$ polyethylene glycol (PEG; tannin inhibitor) per $\mu \mathrm{g}$ CT eliminated up to $87 \%$ of the CT activity $(\mathrm{P}<0.0001)$ compared to incubations without PEG. In conclusion, this study shows that CTs are able to disrupt the life cycle of nematodes and their effects varied according to the parasite species and stage.
\end{abstract}

Keywords: condensed tannins, nematodes, anthelmintic activity, in vitro, bioassays

Gastrointestinal nematodes cause significant production losses in grazing ruminants and their control is achieved mainly by anthelmintics (Vlassof and McKenna 1994). The increased incidence of anthelmintic resistance in farmed sheep, goats and cattle in New Zealand and worldwide, rising consumer concerns about chemical use on farms combined with the finding that regular drenching cannot remove the effects of the parasite entirely (Jackson and Coop 2000, Leathwick et al. 2001), places limitations on the continued use of anthelmintics. Therefore, alternative parasite control strategies are needed.

Some studies have shown that condensed tannins (CTs)containing forages have had significant effects on intestinal nematodes in sheep (Niezen et al. 1995), but they could not determine whether the effect was indirect or direct. Our previous research (Molan et al. 2000a,b) showed for the first time that CTs extracted from a wide range of forages have direct inhibitory effects against the larvae of sheep and deer gastrointestinal nematodes and lungworms in vit$r o$ and have the ability to slowdown the hatching process as well as to prevent the hatched larvae from attaining full development to infective larvae (Molan et al. 2002, Schreurs et al. 2002, Molan and Farag 2010). This may lead to dis- ruption of the parasite life cycle and reduce or prevent pasture contamination with infective larvae.

Radiata pine, Pinus radiata Don (Coniferopsida: Pinaceae), is native to the coast of southern California, USA and Mexico (Millar 1999). Pine bark extract is rich in phenolic compounds such as catechin, epicatechin, quercetin, dihydroquercetin, taxifolin, phenolic acids and procyanidins. It has been used in traditional medicine in Europe and North America for long time for many diseases and illnesses (Packer et al. 1999). Kim et al. (2005) investigated the inhibitory effect of pine bark extract on carbohydrate-hydrolysing enzymes and the hypoglycaemic effect in diabetic mice. The authors found that pine bark extract showed inhibitory effect against salivary $\alpha$-amylase and yeast $\alpha$-glucosidase. Recently, Molan et al. (2009) investigated the effect of purified CTs extracted from pine bark on the sporulation of oocysts of three eimerian species and reported that water-soluble CT extracts have anticoccidial activity as evidenced by their ability to decrease significantly the sporulation of the oocysts of Eimeria tenella (Railliet et Lucet, 1891), E. maxima (Tyzzer, 1929) and E. acervulina (Tyzzer, 1929), under laboratory conditions. 
The CT extracts (especially when obtained from inexpensive sources such as pine bark) may provide another route for reducing parasitism compared to feeding CT-containing forages, which require specialist establishment and management and have a seasonal production (Molan et al. 2009, Min et al. 2012). By applying the phenolic and tannin components in waste pine bark to the animal health sector, the research will in the first instance create the conditions for a new bark processing industry, which will add value to, and also minimise the polluting aspects of, waste products.

The CTs from pine bark has been targeted in this work because the bark is cheap and abundant. The use of waste pine bark in animal health sector represents an ideal combination of using a problem waste from the forestry industry to enhance the production of the farming sector in a significant way. Tannins may affect ecosystem processes by a number of mechanisms including complexation with proteins or metals, inactivation of enzymes, inhibition of microorganisms and plants (Krause et al. 2003). Accordingly, removal of CTs from pine bark would be very desirable for the ecosystem.

In order to investigate whether the CTs from pine bark have direct effects on the nematodes of sheep, a series of in vitro experiments have been conducted to test the effect of CTs on the viability of the eggs and larvae of Trichostrongylus colubriformis (Giles, 1892) and Teladorsagia circumcincta (Stadelmann, 1894) using in vitro bioassays (larval migration inhibition - LMI, egg hatching - EH, and larval development - LD).

\section{MATERIALS AND METHODS}

\section{Preparation of purified condensed tannins}

Pinus radiata bark was obtained from 7-10 year old pine trees grown in Manawatu area, New Zealand. The bark was fed through a wood chipper two times and then ground in a Wiley ${ }^{\mathrm{TM}}$ mill through a $2 \mathrm{~mm}$ mesh. The ground bark was further reduced in size in a Cyclotech (Foss Tecator ${ }^{\mathrm{TM}}$, Sydney, Australia) rotary grinder to yield a fine powder. The finely ground bark was mixed with $70 \%$ acetone $(1: 4)$ containing $1 \mathrm{~g} / 1$ ascorbic acid in a Warring Blender. Acetone extracts were reduced to the aqueous phase by evaporation under reduced pressure at $50^{\circ} \mathrm{C}$. The resulting aqueous phase was mixed with methylene chloride in a separating flask and the upper layer containing the CT was collected. Traces of methylene chloride were removed from the remaining aqueous fraction by rotary evaporation. The resulting fraction was freeze-dried, redissolved in $1: 1$ methanol/ $\mathrm{H}_{2} \mathrm{O}$ $(\mathrm{v} / \mathrm{v})$ and then purified by using a column containing Sephadex LH-20 (Pharmacia, Uppsala, Sweden). The Sephadex LH-20 extracts were freeze-dried and stored at $-20{ }^{\circ} \mathrm{C}$ until required. In all bioassays, the incubations were done with and without the addition of $2 \mu \mathrm{g}$ polyethylene glycol (PEG; tannin inhibitor) per $\mu \mathrm{g}$ of CTs to see if PEG has the ability to inactivate the action of CTs.

\section{Larval migration inhibition (LMI) bioassay}

The larval migration inhibition (LMI) bioassay procedure was used to determine the inhibitory effect of purified CTs against Trichostrongylus colubriformis and Teladorsagia circumcincta (see Molan et al. 2000a). Briefly, the larvae were exsheathed in sodium hypochlorite solution $(0.025 \%$ available chlorine; Rabel et al. 1994), washed five times with tap water and concentrated to 1500 larvae/ml water. The bioassay involved preparation of test solutions with different concentrations of purified CTs $(0,100,200,400,800,1000,1500$ and $2000 \mu \mathrm{g} / \mathrm{ml})$ and of L3 larvae which were combined and incubated in the wells of 48-well tissue culture plates (Costar, Cambridge, MA, USA). The plates were incubated for $2 \mathrm{~h}$ at $37^{\circ} \mathrm{C}$ after which solutions were transferred to sieves ( $7 \mathrm{~mm}$ ID with $20 \mathrm{~m}$ mesh at one end) and left overnight (16-18 h) at room temperature to enable the active larvae to migrate through the sieves for counting. The $20 \mathrm{~m}$ mesh size was selected to ensure that active migration of the larvae through the sieve was determined. The cross-diameter of L3 larvae (25 m; Rabel et al. 1994) is slightly larger than the mesh and would thus prevent the larvae 'falling' through the sieve. Four replicate samples were run for each concentration of $\mathrm{CT}$ as well as negative controls.

\section{Egg hatch bioassay}

The eggs were collected from the fresh faeces of lambs experimentally infected with Te. circumcincta and Tr. colubriformis as described previously (Molan and Farag 2010). This bioassay has been conducted as described previously (Molan et al. 2002). Briefly, a stock solution of CT was prepared by dissolving CT in distilled water. Working solutions of CT were prepared by further dilutions with distilled water. Twenty $\mathrm{ml}$ of the working solutions were pipetted into each well of 48 well tissue culture plates (Costar) together with about 100 eggs and made up to $2 \mathrm{ml}$ with distilled water to give CTs final concentrations ranging from $100 \mu \mathrm{g}$ to $2000 \mu \mathrm{g} / \mathrm{ml}$. Assays were conducted in quadruplicate. Eggs in distilled water alone were used as controls. The eggs were incubated at $24^{\circ} \mathrm{C}$ for $26 \mathrm{~h}$. At the end of incubation a drop of Lugol's iodine was added (to stop further hatching) and the numbers of unhatched eggs and L1 larvae were counted to determine the proportion of eggs hatched (number of L1 larvae/number of eggs in culture $\times 100$ ).

\section{Larval development bioassay}

The assays were carried out in 96-well micro-titre plates (Molan et al. 2004). The assay involved mixing $40 \mathrm{ml}$ of growth medium with $60 \mathrm{ml}$ of larval suspension (containing approximately 100 L1 larvae) and a series of CT concentrations. Duplicate assays were run for each concentration of CTs $(0,25,50$, 100,150 and $200 \mu \mathrm{g} / \mathrm{ml}$ ) and four control wells containing eggs; growth medium but no CTs were included in each experiment. After the addition of CTs, the plates were incubated at $24^{\circ} \mathrm{C}$ for 7 days in a large covered glass Petri dish sealed with paraffin film to maintain a high relative humidity and prevent the plates from drying out (Taylor 1990). At the end of incubation period, a drop of Lugol's iodine was added to each well to stop further development and the numbers of L1, L2, and L3 larvae were counted and the mean larval development was calculated (number of L3 larvae/number of L1 larvae in the medium $\times 100$ ).

\section{Growth medium}

The medium used to culture used by Hubert and Kerboeuf (1984) and modified by Molan et al. (2002) was used to culture the parasite from the $\mathrm{L} 1$ to the third infective larval stage. The culture consisted of a suspension of Escherichia coli plus nutritive medium and amphotericin B. The E. coli suspension was prepared by dissolving $15 \mathrm{mg}$ lyophilised $E$. coli cells (strain W (ATCC) 9637; Sigma, Sydney, Australia) in $100 \mathrm{ml}$ of distilled 
water, and sterilising it by autoclaving. The nutritive medium was prepared by dissolving $1 \mathrm{~g}$ of yeast extract (Y-1000; Sigma) in $90 \mathrm{ml}$ of $0.85 \%$ saline solution plus $10 \mathrm{ml}$ of Earle's balanced salt solution (E75 10; Sigma). The growth medium was prepared by mixing $3 \mathrm{ml}$ of $E$. coli suspension, $3 \mathrm{ml}$ of the nutritive medium and $180 \mu \mathrm{l}$ of amphotericin B (to inhibit the growth of fungi).

\section{Calculation of data and statistical analyses}

The per cent inhibition of egg hatching (EH assay), per cent inhibition of larval development (LD assay) and per cent inhibition of migration (LMI assay) were calculated by using the following equation: $\%$ inhibition $=(\mathrm{A}-\mathrm{B}) / \mathrm{A} \times 100$, where $\mathrm{A}$ is the number of eggs hatched (EH assay), the number of hatched larvae that managed to develop into L3 larvae (LD assay), or the number of L3 larvae migrated through the sieves in the LMI assay in control incubations, and B is the number of eggs hatched, number of L3 larvae in LD assay, or the number of L3 migrated in incubations containing different concentrations of pine bark.

To provide another parameter of comparison between the two nematodes, we calculated the concentration at which $50 \%$ $\left(\mathrm{IC}_{50}\right)$ of the infective larvae failed to pass through the sieves of the LMI assay, the concentration at which $50 \%$ of eggs failed to hatch and the concentration at which $50 \%$ of the hatched larvae failed to achieve full development to $\mathrm{L} 3$ larvae. The $\mathrm{IC}_{50}$ was calculated by using probit analysis and Genstat programme.

Data are presented as the mean \pm standard error of the mean (S.E.M.). A $t$-test or one-way ANOVA, followed by Tukey's test, were used to detect significance among groups and different concentrations. $\mathrm{P}<0.05$ was considered to be statistically significant.

\section{RESULTS}

\section{Concentration of condensed tannins in the bark}

The powdered extract of pine bark used in this study contained $35 \%$ CTs, of which $31 \%$ was free, $3.5 \%$ was protein-bound and $0.5 \%$ was fibre-bound (data not included).

\section{Effect of CT on the motility of the infective L3 larvae}

The present study shows for the first time that watersoluble extracts from pine bark containing 35\% condensed tannins (Molan et al. 2009) have antiparasitic activity as evidenced by the results from the larval migration inhibition (LMI) assay (Fig. 1). The CT extracts showed inhibitory effect against the larvae of both sheep nematodes as evidenced by their ability to inhibit the passage of L3 larvae of these parasites through $20 \mathrm{~m}$ nylon mesh sieves relative to the CT-free control incubations. About $86 \%$ of the larvae in the negative controls (no added CTs) passed through the sieves. At $1000 \mathrm{~g} / \mathrm{ml}$, CTs prevented 48\% and $69 \%$ of Trichostronylus colubriformis and Teladorsagia circumcincta L3 larvae, respectively, from passing through the sieves in comparison with the control incubations with no CTs. Statistical analysis shows that the larvae of Te. circumcincta were more $(\mathrm{P}<0.05)$ susceptible to the effect of CTs than the larvae of Tr. colubriformis.

Addition of $2 \mu \mathrm{g}$ polyethylene glycol (PEG; tannin inhibitor) per $\mu \mathrm{g}$ CT eliminated $65-76 \%$ and $73-82 \%$ of the CT inhibitory activity $(\mathrm{P}<0.0001)$ against the larvae

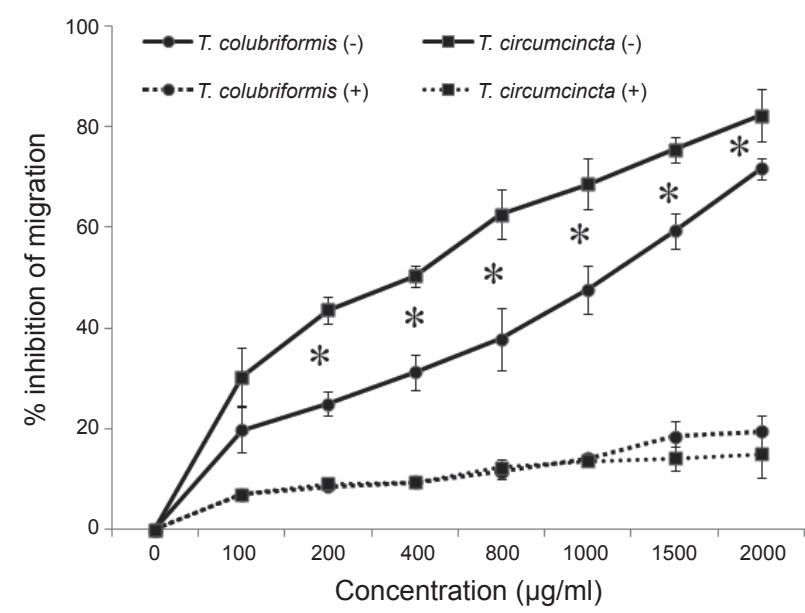

Fig. 1. The effect of condensed tannin (g CT/ml) extracted from pine bark on the motility of the infective third-stage (L3) larvae of Trichostrongylus colubriformis and Teladorsagia circumcincta in vitro. The incubations were undertaken with $(+)$ and without (-) addition of polyethelene glycol (PEG). Each point represents the mean \pm S.E.M. of two independent experiments of duplicate incubations. Asterisks indicate statistically significant differences between the two nematodes $(* \mathrm{P}<0.05-0.001)$.

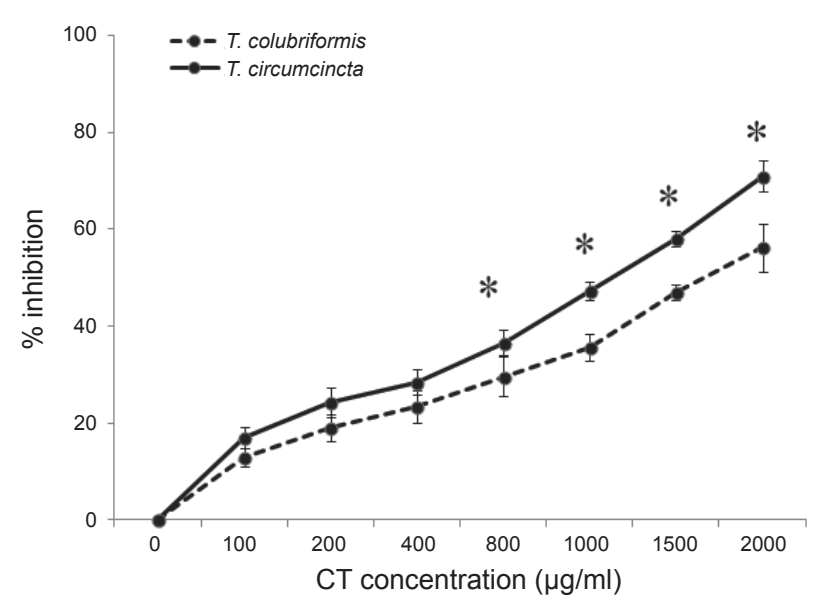

Fig. 2. The effect of condensed tannins (CT) extracted from pine bark on hatching of Trichostrongylus colubriformis and Teladorsagia circumcincta eggs in vitro. Each point represents the mean \pm S.E.M. of two independent experiments of duplicate incubations. Asterisks indicate statistically significant differences between the two nematodes ( $* \mathrm{P}<0.05-0.001)$.

of Te. cirumcincta and Tr. colubriformis, respectively, in comparison with incubations without PEG (Fig. 1).

\section{Effect of CT on the hatching of eggs}

The results of the egg hatch assay are shown in Fig. 2. In control wells (without CTs) about $92 \%$ of the eggs hatched. It can be seen from Fig. 2 that the proportion of egg hatching decreases with increasing CT concentration in both nematodes. When the concentration was $200 \mu \mathrm{g} / \mathrm{ml}$, the proportion of unhatched eggs was $19 \%$ in the incubations containing the eggs of Tr. colubriformis compared to $24 \%$ 


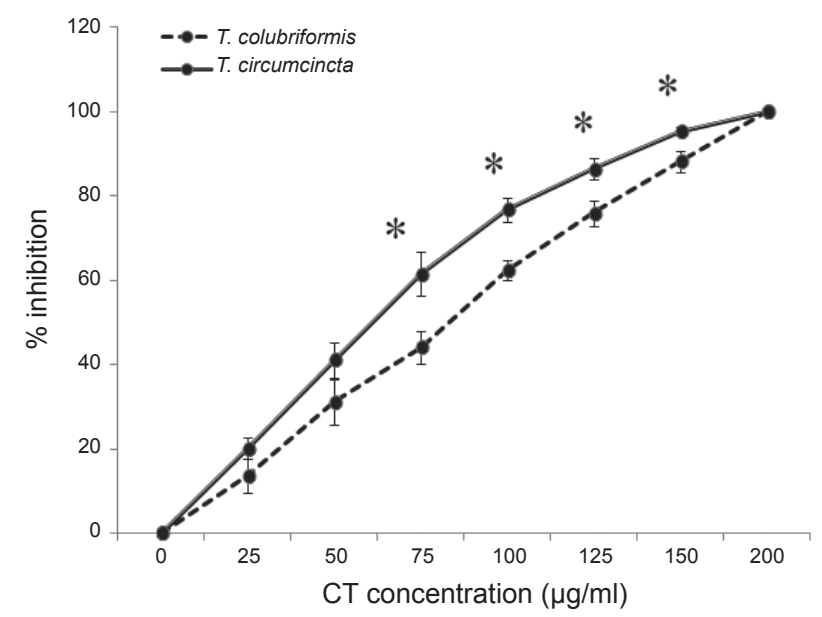

Fig. 3. The effect of pine bark condensed tannins on the development of Trichostrongylus colubriformis and Teladorsagia circumcincta first stage (L1) larvae into infective (L3) larvae. Each point represents the mean \pm S.E.M. of two independent experiments of duplicate incubations. Asterisks indicate statistically significant differences between the two nematodes $(* \mathrm{P}<0.05-0.001)$.

in the incubations containing the eggs of Te. circumcincta. At $1000 \mu \mathrm{g} / \mathrm{ml}$, bark CTs were able to prevent $36 \%$ and $47 \%$ of the eggs of Tr. colubriformis and Te. circumcincta from hatching, respectively, relative to the control incubations without CTs. Eggs of Te. circumcincta were more sensitive $(\mathrm{P}<0.05)$ to the inhibitory effect of pine $\mathrm{CT}$ s than the eggs of $T r$. colubriformis. Addition of $2 \mu \mathrm{g}$ polyethylene glycol (PEG; tannin inhibitor) per $\mu \mathrm{g}$ CT eliminated up to $84 \%$ (data not shown) of the CT inhibitory activity $(\mathrm{P}<0.0001)$ against the larvae of Te. cirumcincta and Tr. colubriformis compared to incubations without PEG.

Effect of CT on the development of first stage (L1) larvae to L3 larvae. In control incubations (without CTs), $88 \%$ of the L1 attained full development to L3 larvae. When the medium contained $100 \mu \mathrm{g} / \mathrm{ml}$, CTs from pine bark prevented $62 \%$ and $77 \%$ of Tr. colubriformis and Te. circumcincta L1 larvae from attaining full development to L3 larvae, respectively in comparison with the control incubations with no CTs. At $200 \mu \mathrm{g} / \mathrm{ml}$, CTs completely inhibited the development of eggs to L3 larvae in both parasites (Fig. 3). Addition of $2 \mu \mathrm{g}$ polyethylene glycol (PEG; tannin inhibitor) per $\mu \mathrm{g}$ CT eliminated up to $87 \%$ (data not shown) of the CT inhibitory activity $(\mathrm{P}<0.0001)$ against the larvae of Te. cirumcincta and Tr. colubriformis compared to incubations without PEG.

From the larval migration inhibition (LMI), egg hatching $(\mathrm{EH})$, and larval development (LD) assays data, the $\mathrm{IC}_{50}$ values were calculated (Table 1). The results show that the eggs, L1 and L3 larvae of Te. circumcincta are significantly more sensitive $(\mathrm{P}<0.0001)$ to the inhibitory effects of pine bark CTs than their counterparts of Tr. colubriformis as evidenced by the significantly lower $(\mathrm{P}<0.0001) \mathrm{IC}_{50}$ values.
Table 1. Concentrations of condensed tannins (CTs) extracted from pine bark that inhibited the passage of $50 \%$ of L3 larvae through the sieves of the larval migration inhibition (LMI) assay, inhibited the hatching of $50 \%$ of eggs, and inhibited the development of $50 \%$ of hatching larvae to infective stage larvae under in vitro conditions $\left(\mathrm{IC}_{50}\right)$. In all bioassays, the $\mathrm{IC}_{50}$ values for Trichostrongylus colubriformis were significantly lower $(\mathrm{P}<0.05-0.0001)$ than those for Teladorsagia circumcincta.

\begin{tabular}{lccc}
\hline & \multicolumn{3}{c}{$\begin{array}{c}\mathrm{IC}_{50} \text { value } \\
(\mu \mathrm{g} / \mathrm{ml}) *\end{array}$} \\
\cline { 2 - 4 } Parasite & LMI & $\begin{array}{c}\text { Egg } \\
\text { hatching }\end{array}$ & $\begin{array}{c}\text { Larval } \\
\text { development }\end{array}$ \\
\hline Trichostrongylus colubriformis & 312 & 390 & 69 \\
Teladorsagia circumcincta & 234 & 320 & 55 \\
\hline * the $\mathrm{IC}_{50}$ was estimated by plotting X-Y and fitting the data with \\
a straight line (line regression) after the data of the X-axis have been \\
logarithm-transformed. $\mathrm{IC}_{50}$ values then estimated using the fitted line \\
equation.
\end{tabular}

\section{DISCUSSION}

The main aim of this study was to investigate the effects of CTs extracted from pine bark on the in vitro viability of infective (L3) larvae of the most important gastrointestinal sheep nematodes, Trichostrongylus colubriformis and Teladorsagia circumcincta. In addition, the effects of the same CTs on the egg hatching and larval development of the two nematodes were also evaluated under in vitro conditions. The CT concentrations used in this study are of a physiological importance as they are within the range of $\mathrm{CT}$ concentrations in the abomasal and duodenal digesta of sheep fed CT-containing forages (Terrill et al. 1994).

The results presented here show that CTs extracted from pine bark have the capacity to inhibit the migration of infective L3 larvae of both Tr. colubriformis and Te. circumcincta under in vitro conditions. This inhibitory activity was attributed to the CTs based on the finding that the addition of polyethylene glycol (PEG) has eliminated up to $87 \%$ of the inhibitory effect of CTs against the larvae. Molan et al. (2000a) investigated the effects of purified CTs from seven forages on the motility of Tr. colubriformis by using the larval migration inhibition (LMI) assay and found that the CTs were able to inhibit up to $63 \%$ of the larvae from passing through the sieves. The authors suggested that these forages may be used as a means to reduce dependence upon proprietary anthelmintics. In comparison with the CTs extracted from the forages tested by Molan et al. (2000a), it seems that CTs extracted from pine bark are more effective in inhibiting the motility of Tr. colubriformis and this may be attributed to the difference in the molecular weight (MW) and chemical composition. It has been reported that CTs from different plant species have different MW and chemical composition of the CTs (Foo et al. 1997, Singh et al. 1997, De Bruyne et al. 1999). Moreover, it has been shown that the inhibitory activities of CTs might be influ- 
enced by the prodelphinidin : procyanidin ratios (Molan et al. 2003) and the degree of polymerisation (Novobilský et al. 2013).

Although it is difficult to explain the reason, the results clearly showed that the L3 larvae of Te. circumcincta were more susceptible to the inhibitory action of pine bark CTs than those of $T r$. colubriformis. Similarly, Paolini et al. (2004) tested the effect of CTs from three woody plants on parasitic nematodes in vitro and reported that Te. circumcincta and Haemonchus contortus (Cobb, 1898) were more susceptible to the inhibitory effects of plant extracts than Tr. colubriformis. Molan et al. (2004) investigated the effect of CTs extracted from sulla (Hedysarum coronarium Koenig) on the viability of three sheep nematodes (Te. circumcincta, H. contortus and Tr. colubriformis) using LMI assay and found that the larvae of Tr. colubriformis were more resistant to the inhibitory effect of CTs than the larvae of the other two nematodes.

It seems that the in vitro assays are useful in determining the effects of extracts and natural products on adult and larval stages of parasites as evidenced by the results of some studies that showed a positive relationship between in vitro and in vivo results. Molan et al. (2000a,b) and Molan and Farag (2010), showed that purified CTs extracted from various forages and medicinal plants reduced the motility of L3 larvae, minimised the rate of egg hatching, and the development of eggs and L1 larvae into infective L3 larvae under in vitro conditions. These results agree well with those observed in ruminants (Athanasiadou et al. 2000a,b, Paolini et al. 2003a,b).

Recently, Min et al. (2012) conducted an in vivo study to determine the effects of ground pine bark (PB) supplementation on animal performance, rumen fermentation, blood variables, faecal egg counts (FEC) and carcass traits in Kiko crossbred male goats and reported that supplementation of $\mathrm{PB}$ reduced FEC, improved performance and favourably modified rumen fermentation. Our in vitro results tend thus to support previous in vivo data obtained with pine bark by Min et al. (2012) and those by Paolini et al. (2003b) who tested the quebracho tannins on the two parasitic stages of Te. circumcinta and Tr. colubriformis.

The results clearly show that nematode larval development is more sensitive to the activity of pine bark CTs than egg hatching as evidenced by the significantly lower $\mathrm{IC}_{50}$ values in the larval development than that in the egg hatching. This may be related to the difference in the length of the exposure time to CTs in the two bioassays.
In the egg hatching assay, eggs were exposed for only one day whereas the in the larval development assay the hatching larvae were exposed for 7 days. Moreover, the egg shell may protect the larvae inside the egg from the harmful effect of CTs in the egg hatching assay whereas the hatched larvae were in direct contact with the CTs in the larval development assay. This trend of activity supports the results of our previous in vitro studies on CTs extracted from different forages (Molan et al. 2002, 2003, Molan and Farag 2010).

The inhibitory effects of pine bark were attributed to the CT contents. The use of polyethylene glycol (PEG) to eliminate effects of CTs was demonstrated by Molan et al. (2004) who showed preferential binding between PEG and CTs relative to protein and CTs under in vitro conditions. Moreover, addition of $2 \mu \mathrm{g}$ PEG per $\mu \mathrm{g}$ CTs to the incubations eliminated up to $93 \%$ of the inhibitory effect of CTs on larval viability through inactivation of CTs (Molan et al. 2000a, 2004). The authors concluded that the elimination of the inhibitory effect of CTs against the larvae in the LMI assay confirms that CTs is the source of the inhibitory action.

Although the mode of action by which CTs affects the eggs and larvae of the nematodes is not precisely known, the failure of the CT-exposed larvae to pass through the sieves may indicate sort of paralysis and interference with neurophysiology or neuromuscular coordination of the larvae (Molan et al. 2000a, 2004). The ability of CTs to bind to proteins and to change their physical and chemical properties (Aerts et al. 1999, Min et al. 2003, Molan et al. 2004) may be another mechanism, due to the fact that nematode cuticle is a proline- and hydroxyprolinerich structure (Thompson and Geary 1995). In support of this hypothesis, Hoste et al. (2006) reported that incubation of adult Tr. colubriformis with chestnut extracts has led to a disruption of the cuticle as shown by scanning electron microscopy after contact with CTs in comparison with the adult worms incubated in phosphate-buffered solution (control).

The efficacy of the low concentrations of CTs used in this study suggests that CTs may offer a potential route for minimising development of eggs into infective larvae under farming conditions. In addition, this study showed clearly that the effects of pine bark CTs varied according to the parasite species and stage.

Acknowledgements. The author would like to thank Mr James Liu for technical support.

\section{REFERENCES}

Aerts R.J., McNabb W.C., Molan A.L., Brand A., Peters J., BARRY T.N. 1999: Condensed tannins from Lotus pedunculatus and Lotus corniculatus effect the degradation of ribulose1,5-bisphosphate carboxylase (Rubisco) protein in the rumen differently. J. Sci. Food Agric. 79: 79-83.
Athanasiadou S., Kyriazakis I., Jackson F., Coop R.L. 2000a: Effects of short term exposure to condensed tannins on adult T. colubriformis. Vet. Rec. 146: 728-732.

Athanasiadou S., Kyriazakis I., Jackson F., Coop R.L. 2000b: Consequences of long-term feeding with condensed 
tannins on sheep parasited with T. colubriformis. Int. J. Parasitol. 30: 1025-1033.

De Bruyne T., Pieters L., Deelstra H., Vlietinck A. 1999: Condensed vegetable tannins: biodiversity in structure and biological activities. Biochem. System. Ecol. 27: 445-459.

Foo L.Y., McNabi W.C., Waghorn G.C., Ulyatt M.J. 1997: Proanthocyanidins from Lotus pedunculatus. Phytochemistry 45: 1689-1696.

Hoste H., Jackson F., Athanasiadou S., Thamsborg S.M., Hoskin S.O. 2006: The effects of tannin-rich plants on parasitic nematodes in ruminants. Trends Parasitol. 22: 254-261.

Hubert J., Kerboeuf D. 1984: A new method for culture of larvae used in diagnosis of ruminant gastrointestinal strongylosis: comparison with faecal cultures. Can. J. Comp. Med. 48: 63-71.

JACKSON F., COOP R.L. 2000: The development of anthelmintic resistance in sheep nematodes. Parasitology 120: 95-107.

Kim Y.M., JeOng Y.K., Wang M.H., Lee W.Y., Rhee H.I. 2005: Inhibitory effect of pine extract on $\alpha$-glucosidase activity and postprandial hyperglycemia. Nutrition 21: 756-761.

Krause T.E.C., Dahlgren R.A., Zasoski R.J. 2003: Tannins in nutrient dynamics of forest ecosystems - a review. Plant Soil 256: 41-66.

Leathwick D.M., Pomroy W.E., Heath A.C.G. 2001: Anthelmintic resistance in New Zealand. N. Z. Vet. J. 49: 227-235.

Millar C.I. 1999: Evolution and biogeography of Pinus radiata, with a proposed revision of its quarternary history. N. Z. J. For. Sci. 29: 335-365.

Min B.R., Barry T.N., Attwood G.T., Mnabb W.C. 2003: The effect of condensed tannins on the nutrition and health of ruminants fed fresh temperate forages: a review. Anim. Feed Sci. Technol. 106: 3-19.

Min B.R., Solaiman S., Gurung N., Behrends J., Eun J., TAHA E., Rose J. 2012: Effects of pine bark supplementation on performance, rumen fermentation, and carcass characteristics of Kiko crossbred male goats. J. Anim. Sci. 90: 3556-3567.

Molan A.L., Alexander R., Brookes I. M., McNabb W.C. 2004: Effects of sulla condensed tannins on the degradation of riblose-1,5-bisphosphate carboxylase/oxygenase (Rubisco) and on the viability of three sheep gastrointestinal nematodes in vitro. J. Anim. Vet. Adv. 3: 165-174.

Molan A.L., Farag A.M. 2010: The effects of condensed tannins extracted from different plant species on egg hatching and larval development of Teladorsagia circumcinicta (Nematoda: Trichostrongylidae). Folia Parasitol. 57: 62-68.

Molan A.L., Hoskin S.O., Barry T. N., McNabb W.C. 2000b: The effect of condensed tannins extracted from four forages on deer lungworm and gastrointestinal nematode larval viability. Vet. Rec. 147: 44-48.

Molan A.L., Liu Z., De S. 2009: Effect of pine bark (Pinus radiata) extracts on sporulation of coccidian oocysts. Folia Parasitol. 56: 1-5.

Molan A.L., Meagher L.P., Spencer P.A., Sivakumaran S. 2003: Effect of flavan-3-ols on in vitro egg hatching, larval development and viability of infective larvae of Trichostrongylus colubriformis. Int. J. Parasitol. 33: 1691-1698.

Molan A.L., Waghorn G.C., McNabb W.C. 2002: Effect of condensed tannins on egg hatching and larval development of Trichostrongylus colubriformis in vitro. Vet. Rec. 150: 65-69.
Molan A.L., Waghorn G.C., Min B.R., McNabb W.C. 2000a: The effect of condensed tannins from seven herbages on Trichostrongylus colubriformis larval migration in vitro. Folia Parasitol. 47: 39-44.

Niezen J.H., Waghorn T.S., Charleston W.A.G., WaGHORN G.C. 1995: Growth and gastrointestinal nematodes parasitism in lambs grazing lucerne (Medicago sativa) or sulla (Hedysarum coronarium) which contains condensed tannins. J. Agric. Sci., Camb. 125: 281-289.

Novobilský A., Stringano E., Hayot Carbonero C., Smith L.M.J., Enemark H.L., Muller-Harvey I., Thamsborg S.M. 2013: In vitro effects of extracts and purified tannins of sainfoin (Onobrychis viciifolia) against two cattle nematodes. Vet. Parasitol. 196: 532-537.

Packer L., Rimbach G., Virgili F. 1999: Antioxidant activity and biologic properties of a procyanidin-rich extract from pine (Pinus maritima) bark, pycnogenol. Free Radic. Biol. Med. 27: 704-724.

Paolini V., Bergeaud J.P., Grisez C., Prevot F., Dorchies P.H, Hoste H. 2003a: Effects of condensed tannins on goats experimentally infected with Haemonchus contortus. Vet. Parasitol. 113: 253-261.

Paolini V., Fouraste I., H. Hoste H. 2004: In vitro effects of three woody plant and sainfoin extracts on two parasitic stages of three parasitic nematode species. Parasitology 129: 69-77.

Paolini V., Frayssines A., De la Farge F., Dorchies P.H., Hoste H. 2003b: Efficacy of condensed tannins on established populations and on incoming larvae of Trichostrongylus colubriformis and Teladorsagia circumcincta in goats. Vet. Res. 34: 331-339.

Rabel B., McGregor R., Douch P.G.C. 1994: Improved bioassy for estimation of inhibitory effects of ovine gastrointestinal mucus and anthelmintics on nematode larval migration. Int. J. Parasitol. 24: 671-676.

Schreurs N.M., Molan A.L., Lopez-Villabos N., Barry T.N., MCNAвв W.C. 2002: Effect of grazing undrenched weaner deer on chicory or perennial ryegrass/white clover pasture on gastrointestinal nematode and lungworm viability. Vet. Rec. 151: 348-353.

Singh S., McCallum J., Gruber M.Y., Towers G.H.N., Muir A.D., Вонм B.A., Koupai-Abyazani M.R., Glass A.D.M. 1997: Biosynthesis of flavan-3-ols by leaf extracts of Onobrychis viciifolia. Phytochemistry 44: 425-432.

TAYLOR M. A. 1990: A larval development test for the detection of anthelmintic resistance in nematodes of sheep. Res. Vet. Sci. 49: 198-202.

Terrill T.H., Waghorn G.C., Wooley D.J., McNabb W.C., BARRY T.N. 1994: Assay and digestion of 14C-labelled condensed tannin in the gastrointestinal tract of sheep. Br. J. Nutr. 72: 467-477.

Thompson D.P., Geary T.G. 1995: The structure and function of helminth surfaces. In: J.J. Marr and M. Muller (Eds.), Biochemistry and Molecular Biology of Parasites. Academic Press, London, UK, pp. 203-232.

Vlasoff A., McKenna P.B. 1994: Nematode parasites of economic importance in sheep in New Zealand. N. Z. J. Zool. 21: $1-8$.

Accepted 23 February 2014 